Int. J. Contemp. Math. Sciences, Vol. 2, 2007, no. 7, 309 - 318

\title{
Normalized Matching Property of a Class of Posets Consisting of Subspaces
}

\author{
Huajun Zhang \\ Department of Applied Mathematics \\ Dalian University of Technology \\ Dalian 116024, P. R. China \\ zhhj1979@yahoo.com.cn
}

\begin{abstract}
Let $V_{n}(q)$ be the $n$-dimensional vector space over the finite field with $q$ elements, and let $T_{1}, T_{2}, \ldots, T_{r+1}$ be $r+1$ subspaces of $V_{n}(q)$ such that $V_{n}(q)=\bigoplus_{i=1}^{r+1} T_{i}$. Assuming that $\operatorname{dim}\left(T_{i}\right)=2$ for $1 \leq i \leq r$, let $\mathcal{F}$ be the collection of all subspaces $S$ 's of $V_{n}(q)$ such that $S=\bigoplus_{i=1}^{r+1}\left(S \cap T_{i}\right)$ and $S$ contains at least one $T_{i}$ for some $1 \leq i \leq r$. In this paper we will prove that $\mathcal{F}$ is $\log$ concave and has the normalized matching property.
\end{abstract}

Keywords: Poset, Subspace lattice, Sperner property, Normalized matching property

Mathematics Subject Classification: 05D05, 06A07

\section{Introduction}

A finite poset $P$ is graded if every maximal chain of $P$ has the same length, denoted by $r(P)$ which is called the rank of $P$. Thus, for each $x \in P$ every maximal chain with $x$ as the top element has the same length, denoted by $r(x)$. Here the length of a chain with $k$ elements is $k-1$. Let $P_{i}$ denote the $i$ th rank of $P$ which consists of all $x \in P$ with $r(x)=i, i=0,1,2, \ldots, r(P)$. A graded poset $P$ with rank $n$ is rank symmetric if $P_{i}=P_{n-i}$ for $0 \leq i \leq n / 2$. It is rank unimodal if $\left|P_{0}\right| \leq\left|P_{1}\right| \leq \cdots \leq\left|P_{k}\right| \geq\left|P_{k+1}\right| \geq \cdots \geq\left|P_{n}\right|$ for some $0 \leq k \leq n$. Similarly, we say $P$ is $\log$ concave if $P_{i}^{2} \geq P_{i-1} P_{i+1}$ for $1 \leq i \leq n-1$.

An antichain in $P$ is a subset of $P$ such that any two elements of this subset are incomparable. Clearly, each rank $P_{i}$ of $P$ is an antichain. The poset $P$ is said to have Sperner property if the maximum size of an antichain equals the maximum of the Whitney numbers $\left|P_{n}\right|$. For $A \subseteq P_{i}$, define $\nabla(A)=\{b \in$ $P_{i+1}: b \geq a$ for some $\left.a \in A\right\}$. We say $P$ has the normalized matching property 
(NM), or is normal if

$$
\frac{|\nabla(A)|}{|A|} \geq \frac{\left|P_{i+1}\right|}{\left|P_{i}\right|} \quad \text { for } \text { all } A \subseteq P_{i}, \quad i=0,1,2, \ldots, r(P)-1 .
$$

We say $P$ has the $L Y M$ property, or is a LYM poset if for every antichain $A$ in $P$, the following inequality holds:

$$
\sum_{i=0}^{r(P)} \frac{\left|A \cap P_{i}\right|}{\left|P_{i}\right|} \leq 1 .
$$

It is well known that the normalized matching property is equivalent to LYM property [9]. From [4] we know the following result.

Theorem 1.1 Suppose that posets $P$ and $Q$ are normal and log concave. Then $P \times Q$ is also normal and $\log$ concave.

Let $C(n, k)$ be the collection of all subsets of an $n$-set $Y$ which intersect a fixed $k$-subset $X$. Then $C(n, k)$ is a natural generalization for the subset lattice. Lih [10] first observed this and showed that $C(n, k)$ has the Sperner property. Griggs [2] further showed that $C(n, k)$ has several strong properties. Let $X_{1}, X_{2}, \ldots, X_{r}$ be $r$ 2-subsets of $Y$, and $H$ be the collections of all subsets of $Y$ which contains at least one $X_{i}$. In [3] Horrocks showed that $H$ is log concave and has the LYM property. In [11] Wang investigated the $q$-analogue of $C(n, k)$ and proved that $C[n, k]$ is sperner and log concave. In this paper we will investigated the $q$-analogue of $H$.

Let $V_{n}(q)$ denote an $n$-dimensional vector space over the finite field with $q$ elements and $L\left(V_{n}(q)\right)$ the lattice of subspaces of $V_{n}(q)$, ordered by inclusion. If there is no confusion, write them as $V$ and $L(V)$ for short. It is well known that $L(V)$ is $\log$ concave and has the normalized matching property. Let $T_{1}, T_{2}, \ldots, T_{r+1}$ be $r+1$ subspaces of $V$ such that $V=\bigoplus_{i=1}^{r+1} T_{i}$. Assuming that $\operatorname{dim}\left(T_{i}\right)=2$ for $1 \leq i \leq r$, let $\mathcal{F}$ be the collection of all subspaces $S$ 's of $V$ such that $S=\bigoplus_{i=1}^{r+1}\left(S \cap T_{i}\right)$ and $S$ contains at least one $T_{i}$ for some $1 \leq i \leq r$, and ordered by inclusion $\mathcal{F}$ is a graded poset. When $T_{r+1}=\{0\}$, we write $\mathcal{F}$ as $\mathcal{F}^{\prime}$.

The main result of this paper is the following theorem.

Theorem $1.2 \mathcal{F}$ is log concave and has the normalized matching property.

Note that if $T_{r+1} \neq\{0\}$, then $\mathcal{F}$ is the product of the two posets $\mathcal{F}^{\prime}$ and $L\left(T_{n+1}\right)$. By Theorem 1.1, to prove the main theorem we only need to prove that $\mathcal{F}^{\prime}$ is log concave and has the normalized matching property. In section 2 we will prove that $\mathcal{F}^{\prime}$ has the normalized matching property and in section 3 we will prove that $\mathcal{F}^{\prime}$ is log concave. In the following, we always assume that $V=\bigoplus_{i=1}^{r} T_{i}$. 


\section{Normalized matching property of $\mathcal{F}^{\prime}$}

As stated in [7], some extremal problems can be considered in a weighted poset $(P, w)$, which is a poset $P$ together with a function ( called a weighted function) $w$ from $P$ into the set of non-negative real numbers. The weight $w(A)$ of a subset $A$ of $P$ is defined by $w(A)=\sum_{a \in A} w(a)$. Every poset $P$ can be considered as a weighted poset $(P, w)$, where $w \equiv 1$, that is, $w(x)=1$ for all $x \in P$. We say $(P, w)$ has the Sperner property if some $P_{i}$ is a maximal weighted antichain of $P$. We say $(P, w)$ has the NM property if

$$
\frac{w(\nabla(A))}{w(A)} \geq \frac{w\left(P_{i+1}\right)}{w\left(P_{i}\right)} \text { for all } A \subseteq P_{i}, i=0,1,2, \ldots, r(P)-1 .
$$

Let $\Gamma$ be a permutation acting on $(P, w)$ which preserves the order relation and the weight on $P$, that is, for every $\gamma \in \Gamma, x \leq y$ in $P$ implies $\gamma(x) \leq \gamma(y)$ , and $w(\gamma(x))=w(x)$. Then we have the quotient poset $\left(P / \Gamma, w_{\Gamma}\right)$, where $P / \Gamma$ consists of the $\Gamma$-orbits ordered as follows: $\Gamma(x) \leq \Gamma(y)$ in $P / \Gamma$ if $x^{\prime} \leq y^{\prime}$ for some $x^{\prime} \in \Gamma(x)$ and $y^{\prime} \in \Gamma(y)$, and the weight function $w_{\Gamma}$ is given by $w_{\Gamma}(\Gamma x)=|\Gamma x| w(x)$. By a theorem due to Kleitman, Edelberg and Lubell [8] (see also [1]) we know that $(P, w)$ has the Sperner property if and only if $\left(P / \Gamma, w_{\Gamma}\right)$ does. The NM version of this result was given as follows.

Theorem 2.1 $[6](P, w)$ has the $N M$ property if and only if $\left(P / \Gamma, w_{\Gamma}\right)$ does.

From these results we see that when study the Sperner property or NM property of a poset, we need only consider its quotient poset, which is generally much smaller than itself. By this approach Wang respectively proved in [5] and [6] that the subgroup lattice $L_{k^{n}}(p)$ of an abelian $p$-group has the Sperner property and has the NM property for sufficiently large prime number $p$.

Let $V=\bigoplus_{i=1}^{r} T_{i}$, and $G L(V)$ denote the general linear group over the $2 r$-dimensional space $V$ consisting of all invertible linear transformations of $V$. Let $H$ be the subgroup of $G L(V)$ such that for any $f \in H, f\left(T_{i}\right)=T_{j}$ for $1 \leq$ $i, j \leq r$. It is obvious that $H \cong G L\left(T_{1}\right) 2 S_{r}$ (the Wreath product of $G L\left(T_{1}\right)$ and $\left.S_{r}\right)$, which consists of all $2 r \times 2 r$ matrices of the form $\operatorname{diag}\left(A_{1}, \ldots, A_{r}\right)\left(\alpha \otimes I_{2}\right)$, where $A_{i}$ 's are $2 \times 2$ invertible matrices, $\alpha$ is a $r \times r$ permutation matrix and $I_{2}$ the $2 \times 2$ identity matrix. It is easy to see that $H$ induces an order preserving permutation group acting on $\mathcal{F}^{\prime}$.

For $0 \leq i_{1} \leq i_{2} \leq \cdots \leq i_{r} \leq 2$, let $\left\langle i_{1}, i_{2}, \ldots, i_{r}\right\rangle$ denote the set of all subspaces $A \in L(V)$ such that $\operatorname{dim}(A)=\sum_{j=1}^{r} i_{j}$ and the both multisets $\left\{\operatorname{dim}\left(A \cap T_{1}\right), \ldots, \operatorname{dim}\left(A \cap T_{r}\right)\right\}$ and $\left\{i_{1}, i_{2}, \ldots, i_{r}\right\}$ are identical. Then $\left\langle i_{1}, i_{2}, \ldots, i_{r}\right\rangle$ is an orbit of $H$ in $\mathcal{F}^{\prime}$ and the quotient poset is $\widehat{\mathcal{F}}^{\prime}=\mathcal{F}^{\prime} / H=$ $\left\{\left\langle i_{1}, i_{2}, \ldots, i_{r-1}, 2\right\rangle: 0 \leq i_{j} \leq 2\right.$, for $\left.1 \leq j \leq r-1\right\}$. The ordering of $\widehat{\mathcal{F}}^{\prime}$ is defined as follows: $\left\langle i_{1}, i_{2}, \ldots, i_{r}\right\rangle \leq\left\langle i_{1}^{\prime}, i_{2}^{\prime}, \ldots, i_{r}^{\prime}\right\rangle$ if $i_{j} \leq i_{j}^{\prime}$ for $1 \leq j \leq r$. Under

this order $\widehat{\mathcal{F}}^{\prime}$ is a graded poset. It is obvious that $\widehat{\mathcal{F}}^{\prime}$ is isomorphic to the 
poset $L(r-1,2)$ which is the set of all partitions $\lambda=\left(\lambda_{1}, \lambda_{2}, \cdots, \lambda_{r-1}\right)$ with $0 \leq \lambda_{1} \leq \lambda_{2} \leq \ldots \leq \lambda_{r-1} \leq 2$, where $\lambda_{i}$ is called the $i$ th part of $\lambda$.

Given a $\mu=\left\langle\mu_{1}, \mu_{2}, \ldots, \mu_{r}\right\rangle \in \widehat{\mathcal{F}}^{\prime}$, by $w(\mu)$ we denote the cardinality of the orbit $\mu$. Then Theorem 2.1 says that $\widehat{\mathcal{F}}^{\prime}$ has the NM property if and only if the weighted poset $\left(\widehat{\mathcal{F}}^{\prime}, w\right)$ employs the NM property. To express the weight $w(\mu)$ we introduce a few of notations.

For any $\lambda=\left\langle\lambda_{1}, \lambda_{2}, \ldots, \lambda_{r}\right\rangle \in \widehat{\mathcal{F}}^{\prime}$, denote $m(\lambda)=\left(m_{0}, m_{1}, m_{2}\right)$, where $m_{j}=m_{j}(\lambda)=\left|\left\{i: \lambda_{i}=j\right\}\right|$. By the definition we immediately have the relation: $r=m_{0}+m_{1}+m_{2}$. For convenience, we use $\widehat{\mathcal{F}}_{s}^{\prime}$ to denote the rank $\left\{\left\langle i_{1}, i_{2}, \ldots, i_{r}\right\rangle: i_{1}+i_{2}+\cdots+i_{r}=s\right\}$.

Define $\{1\}=1,\{i\}=1+q+\cdots+q^{i-1}=\frac{q^{i}-1}{q-1}$ and $\{i\} !=\{1\}\{2\} \cdots\{i\}$. Then $\left[\begin{array}{c}n \\ m\end{array}\right]:=\frac{\{n\} !}{\{n-m\} !\{m\} !}$, the $q$-binomial coefficient, is known for the number of the $m$-subspaces of $V_{n}(q)$.

Lemma 2.2 Let $\lambda=\left\langle\lambda_{1}, \lambda_{2}, \ldots, \lambda_{r}\right\rangle \in \widehat{\mathcal{F}}^{\prime}$ and $m(\lambda)=\left(m_{0}, m_{1}, m_{2}\right)$. Then $w(\lambda)=\left(\begin{array}{c}r \\ m_{0}, m_{1}, m_{2}\end{array}\right)\left[\begin{array}{l}2 \\ 1\end{array}\right]^{m_{1}}$.

Proof. For any $X$ in the orbit of $\lambda$, there are $m_{0} T_{i}^{\prime} s$ such that $T_{i} \cap X=0$, $m_{1} T_{i}^{\prime} s$ such that $\operatorname{dim}\left(T_{i} \cap X\right)=1$ and $m_{2} T_{i}^{\prime} s$ such that $T_{i} \subset X$. It is obvious that there are $\left(\begin{array}{c}r \\ m_{0}, m_{1}, m_{2}\end{array}\right)$ ways to select $T_{i}$ satisfying the above condition. Moreover for each $T_{i}$ there are $\left[\begin{array}{l}2 \\ 1\end{array}\right]$ ways to such that $\operatorname{dim}\left(T_{i} \cap X\right)=1$. Thus $w(\lambda)=\left(\begin{array}{c}r \\ m_{0}, m_{1}, m_{2}\end{array}\right)\left[\begin{array}{l}2 \\ 1\end{array}\right]^{m_{1}}$.

Given $\lambda=\left\langle\lambda_{1}, \lambda_{2}, \ldots, \lambda_{r}\right\rangle \in \widehat{\mathcal{F}}_{k}^{\prime}$ and $m(\lambda)=\left(m_{0}, m_{1}, m_{2}\right)$, we define two order-raising operations $\varphi$ and $\psi$ which map some elements of $\widehat{\mathcal{F}}_{k}^{\prime}$ to $\widehat{\mathcal{F}}_{k+1}^{\prime}$ as follows: $\varphi(\lambda)=\left\langle\lambda_{1}, \lambda_{2}, \ldots, \lambda_{m_{0}}+1, \ldots, \lambda_{r}\right\rangle$ if $m_{0} \geq 1$ and $\psi(\lambda)=$ $\left\langle\lambda_{1}, \lambda_{2}, \ldots, \lambda_{\left(m_{0}+m_{1}\right)}+1, \ldots, \lambda_{r}\right\rangle$ if $m_{1} \geq 1$. Then $m(\psi(\lambda))=\left(m_{0}, m_{1}-1, m_{2}+\right.$ 1) and $m(\varphi(\lambda))=\left(m_{0}-1, m_{1}+1, m_{2}\right)$. It is easy to see that

$$
\nabla(\lambda)=\left\{\begin{array}{cc}
\{\varphi(\lambda), \psi(\lambda)\} & \text { if } m_{0} \geq 1 \text { and } m_{1} \geq 1 \\
\{\varphi(\lambda)\} & \text { if } m_{0} \geq 1 \text { and } m_{1}=0 \\
\{\psi(\lambda)\} & \text { if } m_{0}=0 \text { and } m_{1} \geq 1
\end{array}\right.
$$

Now, we introduce another partial order $\preceq$ on $\widehat{\mathcal{F}}_{s}^{\prime}$ for $1 \leq s \leq 2 r-1$. Let $m(\lambda)=\left(m_{0}, m_{1}, m_{2}\right)$ and $m(\mu)=\left(m_{0}^{\prime}, m_{1}^{\prime}, m_{2}^{\prime}\right)$. We say $\lambda \preceq \mu$ iff $m_{0}+\cdots+$ $m_{i} \leq m_{0}^{\prime}+\cdots+m_{i}^{\prime}$ holds for $i=0,1,2$. Under this partial order $\widehat{\mathcal{F}}_{s}^{\prime}$ is a chain.

Lemma 2.3 Let $\mu, \nu \in \widehat{\mathcal{F}}_{k}^{\prime}$ and $\mu \preceq \nu$. Suppose $m(\mu)=\left(m_{0}, m_{1}, m_{2}\right)$ and $m(\nu)=\left(m_{0}^{\prime}, m_{1}^{\prime}, m_{2}^{\prime}\right)$. Then we have 
(i) If $m_{0} \geq 1, \frac{w\{\varphi(\mu)\}}{w(\mu)}<\frac{w\{\varphi(\nu)\}}{w(\nu)}$;

(ii) If $m_{1}^{\prime} \geq 1, \frac{w\{\psi(\mu)\}}{w(\mu)}>\frac{w\{\psi(\nu)\}}{w(\nu)}$.

Proof. If $m_{0} \geq 1$, set $m_{0}^{\prime}=m_{0}+1, m_{1}^{\prime}=m_{1}-2$ and $m_{2}^{\prime}=m_{1}+1$. Then we obtain

$$
\frac{w(\varphi(\mu))}{w(\mu)} / \frac{w(\varphi(\nu))}{w(\nu)}=\frac{m_{0}}{m_{1}+1} \cdot \frac{m_{1}-1}{\left(m_{0}+1\right)}<1 .
$$

Thus the statement (i) follows by induction. If $m_{1}^{\prime} \geq 1$, we can prove $\frac{w\{\psi(\mu)\}}{w(\mu)}>$ $\frac{w\{\psi(\nu)\}}{w(\nu)}$ in a similar way.

The following trivial result is used repeatedly in the proof.

Lemma 2.4 Let $A_{i}, B_{j}>0$ for $1 \leq i, j \leq k$ and $A_{i} / B_{i} \geq A_{1} / B_{1} \geq \ell$ for $2 \leq i \leq k$. Then

$$
\frac{A_{2}+\cdots+A_{k}}{B_{2}+\cdots+B_{k}} \geq \frac{A_{1}+A_{2}+A_{3}+\cdots+A_{k}}{B_{1}+B_{2}+B_{3}+\cdots+B_{k}} \geq \ell .
$$

Suppose $\widehat{\mathcal{F}}_{s}^{\prime}=\left\{\mu_{1}, \mu_{2}, \ldots, \mu_{k}\right\}$ with $\mu_{1} \preceq \cdots \preceq \mu_{k}$. For $1 \leq i \leq k$, define $S_{i}=\left\{\mu_{i}, \ldots, \mu_{k}\right\}$ and $R_{i}=\left\{\mu_{1}, \ldots, \mu_{i}\right\}$. Thus we immediately have that $w\left(S_{i}\right)$ 's decrease monotonic with $i$ and $w\left(R_{i}\right)$ 's increase monotonic with $i$.

Lemma 2.5 For any rank $\widehat{\mathcal{F}}_{s}^{\prime}$ of $\widehat{\mathcal{F}}^{\prime}$ (where $\left.s<2 r\right)$. Let $\widehat{\mathcal{F}}_{s}^{\prime}=\left\{\mu_{1}, \mu_{2}, \cdots, \mu_{k}\right\}$ and suppose $\mu_{1} \preceq \mu_{2} \preceq \cdots \preceq \mu_{k}$ where $r=\left|\widehat{\mathcal{F}}_{s}^{\prime}\right|$. Then we have:

(i) $\frac{w\left(\nabla\left(S_{k}\right)\right)}{w\left(S_{k}\right)} \geq \frac{w\left(\nabla\left(S_{k-1}\right)\right)}{w\left(S_{k-1}\right)} \geq \cdots \geq \frac{w\left(\nabla\left(S_{1}\right)\right)}{w\left(S_{1}\right)}=\frac{w\left(\hat{\mathcal{F}}_{s+1}^{\prime}\right)}{w\left(\hat{\mathcal{F}}_{s}^{\prime}\right)}$;

(ii) $\frac{w\left(\nabla\left(R_{1}\right)\right)}{w\left(R_{1}\right)} \geq \frac{w\left(\nabla\left(R_{2}\right)\right)}{w\left(R_{2}\right)} \geq \cdots \geq \frac{w\left(\nabla\left(R_{k}\right)\right)}{w\left(R_{k}\right)}=\frac{w\left(\hat{\mathcal{F}}_{s+1}^{\prime}\right)}{w\left(\hat{\mathcal{F}}_{s}^{\prime}\right)}$.

Proof. Since $s=2,2 r-1,2 r, \widehat{\mathcal{F}}_{s}^{\prime}$ has only one element. Then we only need to consider $3 \leq s \leq 2 r-3$. Given $m\left(\mu_{1}\right)=\left(m_{0}, m_{1}, m_{2}\right)$. It is easy to see that

$$
\nabla\left(S_{i}\right)= \begin{cases}\nabla\left(S_{i+1}\right) \cup \varphi\left(\mu_{i}\right), & i \geq 2 \text { or } i=1 \text { and } m_{0} \geq 1 \\ \nabla\left(S_{i+1}\right), & i=1 \text { and } m_{0}=0 .\end{cases}
$$

We thus obtain by Lemma 2.3 and Lemma 2.4 that $\frac{w\left(\nabla\left(S_{i}\right)\right)}{w\left(\nabla\left(S_{i}\right)\right)}=\frac{w\left(\psi\left(\mu_{i}\right)\right)+w\left(\nabla\left(S_{i+1}\right)\right)}{w\left(\mu_{i}\right)+w\left(S_{i+1}\right)}$ $\leq \frac{w\left(\nabla\left(S_{i+1}\right)\right)}{w\left(\nabla\left(S_{i+1}\right)\right)}$ holds for $i \geq 2$ or $i=1$ while $m_{0} \geq 1$. If $i=1$ and $m_{0}=0$, then $S_{1}=\left\{\mu_{1}\right\} \cup S_{2}$ and $\nabla\left(S_{1}\right)=\nabla\left(S_{2}\right)$, so $\frac{w\left(\nabla\left(S_{1}\right)\right)}{w\left(S_{1}\right)}=\frac{w\left(\nabla\left(S_{2}\right)\right)}{w\left(\mu_{1}\right)+w\left(S_{2}\right)}<\frac{w\left(\nabla\left(S_{2}\right)\right)}{w\left(S_{2}\right)}$. Thus the statement (i) follows. Similarly, for $i \leq k$, we have

$$
\nabla\left(R_{i}\right)= \begin{cases}\nabla\left(R_{i-1}\right) \cup \psi\left(\mu_{i}\right), & i<k-1 \text { or } i=k \text { and } s \text { is odd; } \\ \nabla\left(R_{i-1}\right), & i=k \text { and } s \text { is even. }\end{cases}
$$

Then statement (ii) follows by Lemma 2.3 and Lemma 2.4 
Lemma 2.6 Let $\mu_{i}, \mu_{i+1}, \ldots, \mu_{j}$ be the elements of $\widehat{\mathcal{F}}_{s}^{\prime}$ where $1 \leq i \leq j \leq k$. Then $\frac{w\left\{\nabla\left(\mu_{i}, \ldots, \mu_{j}\right)\right\}}{w\left\{\mu_{i}, \ldots, \mu_{j}\right\}} \geq \frac{w\left(\widehat{\mathcal{F}}_{s+1}^{\prime}\right)}{w\left(\widehat{\mathcal{F}}_{s}^{\prime}\right)}$.

Proof. By definition we have that $M=S_{i} \cap R_{j}$ and $\nabla(M)=\nabla\left(S_{i}\right) \cap \nabla\left(R_{j}\right)$. It is easy to see that $S_{i} \cup R_{j}=\widehat{\mathcal{F}}_{s}^{\prime}$ and $\nabla\left(S_{i}\right) \cup \nabla\left(R_{j}\right)=\widehat{\mathcal{F}}_{s+1}^{\prime}$, which implies that $w(M)=w\left(S_{i}\right)+w\left(R_{j}\right)-w\left(\widehat{\mathcal{F}}_{s}^{\prime}\right)$ and $w(\nabla(M))=w\left(\nabla\left(S_{i}\right)\right)+w\left(\nabla\left(R_{j}\right)\right)-$ $w\left(\widehat{\mathcal{F}}_{s+1}^{\prime}\right)$. We thus obtain that

$$
\frac{w(\nabla(M))}{w(M)}=\frac{w\left(\nabla\left(S_{i}\right)\right)+w\left(\nabla\left(R_{j}\right)\right)-w\left(\widehat{\mathcal{F}}_{s+1}^{\prime}\right)}{w\left(S_{i}\right)+w\left(R_{j}\right)-w\left(\widehat{\mathcal{F}}_{s}^{\prime}\right)} \geq \frac{w\left(\widehat{\mathcal{F}}_{s+1}^{\prime}\right)}{w\left(\widehat{\mathcal{F}}_{s}^{\prime}\right)} .
$$

The last inequality follows from Lemma 2.4 and Lemma 2.5.

In order to complete the proof of that $\widehat{\mathcal{F}}^{\prime}$ has the normalized matching property it suffices to prove that for any $1 \leq s \leq 2 r-1, \frac{w(\nabla(M))}{w(M)} \geq \frac{w\left(\hat{\mathcal{F}}_{s+1}^{\prime}\right)}{w\left(\hat{\mathcal{F}}_{s}^{\prime}\right)}$ holds for every $M \subseteq \widehat{\mathcal{F}}_{s}^{\prime}$.

Given a subset $M$ of $\overline{\mathcal{F}}_{s}^{\prime}$, set $M=M_{1} \cup \cdots \cup M_{\ell}(\ell \geq 1)$, where $M_{t}=$ $\left\{\mu_{i_{t}}, \mu_{i_{t}+1}, \ldots, \mu_{j_{t}}\right\}(t=1,2, \ldots, \ell), 1 \leq i_{1} \leq j_{1}<i_{2} \leq j_{2}<\cdots<i_{\ell} \leq j_{\ell} \leq k$. Then $M_{i} \cap M_{j}=\emptyset$ and $\nabla\left(M_{i}\right) \cap \nabla\left(M_{j}\right)=\emptyset$ for $i \neq j$. We thus obtain that $w(M)=w\left(M_{1}\right)+\cdots+w\left(M_{\ell}\right)$ and $w(\nabla(M))=w\left(\nabla\left(M_{1}\right)\right)+\cdots+w\left(\nabla\left(M_{\ell}\right)\right)$. Then the inequality $\frac{w(\nabla(M))}{w(M)} \geq \frac{w\left(\hat{\mathcal{F}}_{s+1}^{\prime}\right)}{w\left(\hat{\mathcal{F}}_{s}^{\prime}\right)}$ follows from Lemma 2.4 and Lemma 2.6 .

\section{$3 \quad$ Log concavity of $\mathcal{F}^{\prime}$}

We need some definitions before proving the result. For each $X \in \mathcal{F}^{\prime}$, let $\partial X=\left(a_{1}, a_{2}, \ldots, a_{r}\right)$ where $a_{i}=\operatorname{dim}\left(X \cap T_{i}\right)$. For any $X, Y \in \mathcal{F}^{\prime}$, suppose $\partial X=\left(a_{1}, a_{2}, \ldots, a_{r}\right)$ and $\partial Y=\left(b_{1}, b_{2}, \ldots, b_{r}\right)$, we define

$$
X \odot Y=\left(C_{1}, C_{2}, \ldots, C_{r}\right),
$$

where $C_{i}$ be the multiset $\left\{X \cap T_{i}, Y \cap T_{i}\right\}$ if $a_{i}+b_{i} \neq 2$, otherwise $C_{i}$ is the subspace spanned by $X \cap T_{i}$ and $Y \cap T_{i}$. Let $S=\left\{X \odot Y: X, Y \in \mathcal{F}^{\prime}\right\}$, and for each $A=X \odot Y \in S$, let $A_{(i, m-i)}=\left\{(X, Y): X \in \mathcal{F}_{i}^{\prime}, Y \in \mathcal{F}_{m-i}^{\prime}\right.$ and $\left.X \odot Y=A\right\}$ where $m=\operatorname{dim}(X)+\operatorname{dim}(Y)$. Clearly, we have $\left|A_{(i, m-i)}\right|=\left|A_{(m-i, i)}\right|$.

To prove that $\mathcal{F}^{\prime}$ is log concave, we only need to prove that $\mathcal{F}_{i-1}^{\prime} \times \mathcal{F}_{i+1}^{\prime} \leq$ $\mathcal{F}_{i}^{\prime 2}$. It is not difficult to see that

$$
\mathcal{F}_{i}^{\prime} \times \mathcal{F}_{j}^{\prime}=\sum_{\substack{A=X \odot Y \in S \\ \operatorname{dim}(X)+\operatorname{dim}(Y)=i+j}}\left|A_{(i, j)}\right| .
$$

Thus the unimodality of $\left\{\left|A_{(i, m-i)}\right|\right\}$ on $i$ implies the log concavity of $\mathcal{F}^{\prime}$. So it remains to verify the unimodality of $\left|A_{(i, m-i)}\right|$. 
Let $B_{A}$ be the multiset $\left\{a_{1}+b_{1}, \cdots, a_{r}+b_{r}\right\}$, we call that $A$ is of type 0 if $4 \in B_{A}$ and $1 \notin B_{A}$; of type 1 if $B_{A}$ only contains 2 or 3 besides 0 ; of type 2 if $B_{A}$ only contains 2 and 3 besides 0 ; of type 3 if $1 \in B_{A}$. It is obviously that for any $A \in S, B_{A}$ must be one type of the above. Suppose $a_{i}+b_{i}=2$ in $B_{A}$ for some $i$, if $C_{i} \neq T_{i}$, then $X \odot Y=A$ implies that $C_{i}=X \cap T_{i}=Y \cap T_{i}$. Thus we only need to consider the case $C_{i}=T_{i}$ when $a_{i}+b_{i}=2$ in $B_{A}$.

Let $T_{(k, i)}=\left\{(X, Y): X \odot Y=\left(T_{1}, \ldots, T_{k}\right), X=\bigoplus_{i=1}^{k}\left(X \cap T_{i}\right), \operatorname{dim}(X)=\right.$ $i$ and $\left.Y=\bigoplus_{i=1}^{k}\left(Y \cap T_{i}\right)\right\}$, we have the following result.

Lemma 3.1 For each integer $k>0$, we have $\left|\left\{T_{(k, i)}\right\}\right|$ is symmetric and unimodal.

Proof. It is obvious that $\left|\left\{T_{(k, i)}\right\}\right|$ is symmetric. By induction on $k$, we are to prove that $\left|\left\{T_{(k, i)}\right\}\right|$ is unimodal.

If $k=1$, then $\left|T_{(1,1)}\right|=q(q+1)>\left|T_{(1,0)}\right|=\left|T_{(1,2)}\right|=1$. Assume that the result is true for $k$ and we consider the case of $k+1$. It is easy to see that

$$
\left|T_{(k+1, i)}\right|=\left|T_{(k, i)}\right|+q(q+1)\left|T_{(k, i-1)}\right|+\left|T_{(k, i-2)}\right| .
$$

Then $\left|T_{(k+1, i+1)}\right|-\left|T_{(k+1, i)}\right|$

$$
\begin{aligned}
= & \left(\left|T_{(k, i+1)}\right|+q(q+1)\left|T_{(k, i)}\right|+\left|T_{(k, i-1)}\right|\right) \\
& -\left(\left|T_{(k, i)}\right|+q(q+1)\left|T_{(k, i-1)}\right|+\left|T_{(k, i-2)}\right|\right) \\
= & \left(\left|T_{(k, i+1)}\right|-\left|T_{(k, i)}\right|\right)+q(q+1)\left(\left|T_{(k, i)}\right|-\left|T_{(k, i-1)}\right|\right)+\left(\left|T_{(k, i-1)}\right|-\left|T_{(k, i-2)}\right|\right) .
\end{aligned}
$$

By the hypothesis of induction and the symmetry of $\left\{T_{(k, i)}\right\}$ we have that $\left|T_{(k+1, i+1)}\right|>\left|T_{(k+1, i)}\right|$ if $0 \leq i \leq k$. Thus the result is true for $k+1$ and we complete the proof.

Lemma 3.2 Let $A=X \odot Y \in S$ and $m=\operatorname{dim}(X)+\operatorname{dim}(Y)$. If $A$ is of type 0 , then $\left|A_{(2, m-2)}\right| \leq\left|A_{(3, m-3)}\right| \leq \cdots \leq\left|A_{(\ell, m-\ell)}\right|$, where $\ell=\lfloor m / 2\rfloor$.

Proof. Suppose $B_{A}$ contains $s 3^{\prime} s$, and we use $A_{(i, m-i)}^{s}$ to replace $A_{(i, m-i)}$. It is easy to see that

$$
\left|A_{(i, m+3-i)}^{s+1}\right|=\left|A_{(i-1, m-i+1)}^{s}\right|+\left|A_{(i-2, m-i+2)}^{s}\right| .
$$

Now we use induction on $s$ to prove the result. By Lemma 3.1 we know that the result is true for $s=0$. Assume that the result is true for $s$ and consider the case of $s+1$. By (4) we have

$$
\left|A_{(i+1, m+2-i)}^{s+1}\right|-\left|A_{(i, m+3-i)}^{s+1}\right|=\left|A_{(i, m-i)}^{s}\right|-\left|A_{(i-2, m-i+2)}^{s}\right| .
$$

If $i \leq\lfloor(m+1) / 2\rfloor$, by the hypothesis of induction and the symmetry of $\left|A_{(i, m-i)}\right|$ on $i$ we have $\left|A_{(i+1, m+2-i)}^{s+1}\right|-\left|A_{(i, m+3-i)}^{s+1}\right| \geq 0$. Thus the result is true for $s+1$ and we derive the result. 
Lemma 3.3 Let $A=X \odot Y \in S$ and $m=\operatorname{dim}(X)+\operatorname{dim}(Y)$. If $A$ is of type 1 , then $\left|A_{(2, m-2)}\right| \leq\left|A_{(3, m-3)}\right| \leq \cdots \leq\left|A_{(k, m-k)}\right|$, where $k=\lfloor m / 2\rfloor$.

Proof. Now we distinguish two cases to consider $B_{A}$.

Case 1. $B_{A}=\{3\}$. Suppose $B_{A}$ contains $k 3^{\prime} s$. It is easy to see that $\left|A_{(k+i, m-k-i)}\right|=\left(\begin{array}{c}k \\ i\end{array}\right)$. By the unimodality of binomial coefficients the statement follows.

Case 2. $B_{A}=\{2\}$. Suppose $B_{A}$ contains $k 2^{\prime} s$. It is easy to see that $A_{(i, k)}$ has the following recursion:

$\left|A_{(i, 2(k+1)-i)}\right|= \begin{cases}\left|T_{(k, i-2)}\right|+q(q+1)\left|A_{(i-1,2 k-i+1)}\right|+\left|A_{(i, 2 k-i)}\right| & \text { if } i<k+1, \\ 2\left|T_{(k, k-1)}\right|+q(q+1)\left|A_{(k, k)}\right| & \text { if } i=k+1,\end{cases}$

where $\left|A_{(i, 2 k-i)}\right|=0$ if $i<2$.

Now, we use induction on $k$ to prove that $\left|A_{(2,2 k-2)}\right| \leq\left|A_{(3,2 k-3)}\right| \leq \cdots \leq$ $\left|A_{(k, k)}\right|$.

If $k=2$, the statement is easy to verify. Assume that the result is true for $k$ and consider the case of $k+1$.

If $i<k+1$, we have $\left|A_{(i, 2 k+2-i)}\right|-\left|A_{(i-1,2 k+3-i)}\right|$

$$
\begin{aligned}
= & \left(\left|T_{(k, i-2)}\right|+q(q+1)\left|A_{(i-1, k)}\right|+\left|A_{(i, 2 k-i)}\right|\right) \\
& -\left(\left|T_{(k, i-2)}\right|+q(q+1)\left|A_{(i-2,2 k+2-i)}\right|+\left|A_{(i-1,2 k-i+1)}\right|\right) \\
= & \left(\left|T_{(k, i-2)}\right|-\left|T_{(k, i-3)}\right|\right)+q(q+1)\left(\left|A_{(i-1, k+1-i)}\right|-\left|A_{(i-2,2 k+2-i)}\right|\right) \\
& +\left(\left|A_{(i, 2 k-i)}\right|-\left|A_{(i-1,2 k-i+1)}\right|\right) \geq 0 .
\end{aligned}
$$

Since each of the three terms in the sum are nonnegative by induction and the unimodality of binomial coefficients.

$$
\text { If } \begin{aligned}
& i=k+1,\left|A_{(k+1, k+1)}\right|-\left|A_{(k, k+2)}\right| \\
&=\left(2\left|T_{(k, k-1)}\right|+q(q+1)\left|A_{(k, k)}\right|\right) \\
&-\left(\left|T_{(k, k-2)}\right|+q(q+1)\left|A_{(k-1, k+1)}\right|+\left|A_{(k, k)}\right|\right) \\
&=\left(\left|T_{(k, k-1)}\right|-\left|T_{(k, k-2)}\right|\right)+q(q+1)\left(\left|A_{(k, k)}\right|-\left|A_{(k-1, k+1)}\right|\right) \\
&+\left(\left|T_{(k, k-1)}\right|-\left|A_{(k-1, k+1)}\right|\right) .
\end{aligned}
$$

It is obvious that $\left|T_{(k, k-1)}\right| \geq\left|A_{(k-1, k+1)}\right|$ when $B_{A}$ only contains 2 besides 0 . Then by induction we have $\left|A_{(k+1, k+1)}\right|-\left|A_{(k, k+2)}\right| \geq 0$, as desired.

For each $A \in S$ and be type 2, suppose $B_{A}$ contains $s$ 's and $k$ 3's, let $A_{(i, k, s)}^{\prime}=\left\{(X, Y): X \in \mathcal{F}_{i}, Y \in \mathcal{F}_{m-i}\right.$ and $\left.X \odot Y=A\right\}$ such that if $a_{i}+b_{i}=2$ in $B_{A}$, then either $T_{i} \subseteq X$ or $T_{i} \cap X=\emptyset$.

Lemma 3.4 Let $A \in S$ and be of type 2 , then $\left|A_{(2, k, s)}^{\prime}\right| \leq\left|A_{(3, k, s)}^{\prime}\right| \leq \cdots \leq$ $\left|A_{(r, k, s)}^{\prime}\right|$ where $r=\lfloor 2 s+3 k / 2\rfloor$. 
Proof. It is easy to see that $\left|A_{(k+1, k, s)}^{\prime}\right|=k,\left|A_{(k, k, s)}^{\prime}\right|=1$ and $\left|A_{(i, k, s)}^{\prime}\right|=0$ for $i<k$. At first, we give a recursion of $\left|A_{(i, k+1, s)}^{\prime}\right|$ :

$$
\left|A_{(i, k+1, s)}^{\prime}\right|= \begin{cases}k+1 & \text { if } i=k+2 \\ \left|A_{(i-2, k, s)}^{\prime}\right|+\left|A_{(i-1, k, s)}^{\prime}\right| & \text { if } k+3 \leq i\end{cases}
$$

In fact, suppose $a_{j}+b_{j}=3$, for $i \geq k+3$ and each $(X, Y) \in A_{(i, 3 k+3+2 s-i)}^{\prime}$. Then we have $\left(X / T_{j}, Y / T_{j}\right) \in A_{(i-1,3 k+2 s+1-i)}^{\prime}$ if $\operatorname{dim}\left(T_{j} \cap X\right)=1$, and $\left(X / T_{j}, Y / T_{j}\right) \in$ $A_{(i-2,3 k+2 s+2-i)}^{\prime}$ otherwise. Thus $\left|A_{(i, k+1, s)}^{\prime}\right|=\left|A_{(i-2, k, s)}^{\prime}\right|+\left|A_{(i-1, k, s)}^{\prime}\right|$.

Now we use induction on $k$ to prove that

$$
\left|A_{(2, k, s)}^{\prime}\right| \leq\left|A_{(3, k, s)}^{\prime}\right| \leq \cdots \leq\left|A_{(r, k, s)}^{\prime}\right| .
$$

If $k=0$, by Lemma 3.3 the result is true. Assume that the result is true for $k$ and we consider the case of $k+1$. If $k+3 \leq i \leq\lfloor(3 k+2 s+3) / 2\rfloor$, $\left|A_{(i, k+1, s)}^{\prime}\right|-\left|A_{(i-1, k+1, s)}^{\prime}\right|$

$$
\begin{aligned}
& =\left(\left|A_{(i-2, k, s)}^{\prime}\right|+\left|A_{(i-1, k, s)}^{\prime}\right|\right)-\left(\left|A_{(i-3, k, s)}^{\prime}\right|-\left|A_{(i-2, k+1, s)}^{\prime}\right|\right) \\
& =\left|A_{(i-1, k, s)}^{\prime}\right|-\left|A_{(i-3, k, s)}^{\prime}\right| .
\end{aligned}
$$

Then by the induction hypothesis and the symmetry of $A_{(i, k, s)}$ on $i$ we have $\left|A_{(i, k+1, s)}^{\prime}\right|-\left|A_{(i-1, k+1, s)}^{\prime}\right| \geq 0$ and the statement follows.

Lemma 3.5 Let $A=X \odot Y \in S$ and $m=\operatorname{dim}(X)+\operatorname{dim}(Y)$. If $A$ is of type 2 , then $\left|A_{(2, m-2)}\right| \leq\left|A_{(3, m-3)}\right| \leq \cdots \leq\left|A_{(k, m-k)}\right|$, where $k=\lfloor m / 2\rfloor$.

Proof. Suppose $B_{A}$ contains $k 3^{\prime} s$ and $s 2^{\prime} s$. For $0 \leq t \leq s$, define

$$
A_{(i, k, t)}=\left\{(X, Y): X \in \mathcal{F}_{i}, Y \in \mathcal{F}_{m-i} \text { and } X \odot Y=A\right\}
$$

such that there are $t T_{i}^{\prime} s$ either $T_{i} \subseteq X$ and $T_{i} \cap Y=\{0\}$ or $T_{i} \subseteq Y$ and $T_{i} \cap X=\{0\}$. By lemma 3.4, we have $\left|A_{(2, k, t)}\right| \leq\left|A_{(3, k, t)}\right| \leq \cdots \leq\left|A_{(\lfloor m / 2\rfloor, k, t)}\right|$. Since $\left|A_{(i, m-i)}\right|=\sum_{t=0}^{s}\left|A_{(i, k, t)}\right|$, then we have $\left|A_{(2, m-2)}\right| \leq\left|A_{(3, m-3)}\right| \leq \cdots \leq$ $\left|A_{(\lfloor m / 2\rfloor,\lceil m / 2\rceil)}\right|$. Thus we complete the proof.

Lemma 3.6 Let $A=X \odot Y \in S$ and $m=\operatorname{dim}(X)+\operatorname{dim}(Y)$. If $A$ is of type 3 , then $\left|A_{(2, m-2)}\right| \leq\left|A_{(3, m-3)}\right| \leq \cdots \leq\left|A_{(k, m-k)}\right|$, where $k=\lfloor m / 2\rfloor$.

Proof. Suppose $B_{A}$ contains $k 1^{\prime} s$, we use $A_{(k, i, m)}$ to denote $A_{(i, m)}$. Now we use induction on $k$ to prove the result. It is easy to see that $\left|A_{(k+1, i, m+1)}\right|=$ $\left|A_{(k, i, m)}\right|+\left|A_{(k, i-1, m)}\right|$. If $k=0$, the result holds true by Lemmas 3.2, 3.3 and 3.5. Assume that the result holds for $k$ and consider the case of $k+1$. For $2 \leq i \leq\lfloor m-1 / 2\rfloor$,

$$
\begin{aligned}
& \left|A_{(k+1, i+1, m+1)}\right|-\left|A_{(k+1, i, m+1)}\right| \\
= & \left(\left|A_{(k, i+1, m)}\right|+\left|A_{(k, i, m)}\right|\right)-\left(\left|A_{(k, i, m)}\right|+\left|A_{(k, i-1, m)}\right|\right) \\
= & \left|A_{(k, i+1, m)}\right|-\left|A_{(k, i-1, m)}\right| .
\end{aligned}
$$


By the induction hypothesis and the symmetry of $\left|A_{(k, i, m)}\right|$ on $i$ we have (6) $\geq 0$ and the statement follows.

By Lemma 3.2-3.6, we know that for any $A=X \odot Y \in S$ and $\operatorname{dim}(X)+$ $\operatorname{dim}(Y)=2 i,\left|A_{(i-1, i+1)}\right| \leq\left|A_{(i, i)}\right|$. Thus we obtain

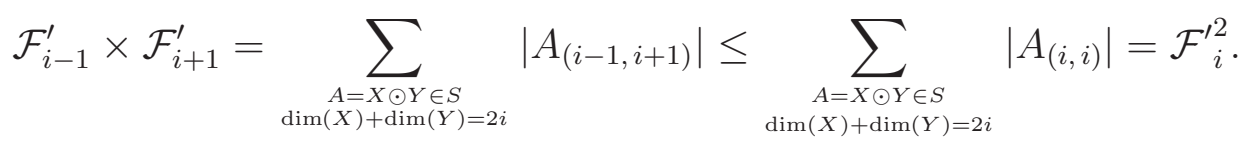

Then the statement follows.

\section{References}

[1] R. Freese, An application of Dilworth's lattice of maximal antichains, Discrete Math., 7 (1974), 107-109.

[2] J.R. Griggs, Collections of subsets with the sperner property, Trans. Amer. Math. Soc., 269 (1982), 575-591.

[3] D.G.C. Horrocks, Filters of the Boolean Algebra having the LYM property, Technical Report CORR 96-09, University of Waterloo, 1996.

[4] W.N. Hsieh and D. Kleitman, Normalized matching in direct products of partial orders, Stud. Appl. Math., 52 (1973), 285-289.

[5] J. Wang, Proof of a conjecture on the sperner property of the subgroup lattice of an abelian p-group, Ann. of Combin., 2 (1998), 85-101.

[6] J. Wang, Normalized matching property of the subgroup lattice of an abelian p-group, Discrete Math., 257 (2002), 559-574.

[7] K. Engel, Sperner theory, Cambridge University Press, Cambridge, 1997. p. 160.

[8] D. Kleitman, M. Edelberg, D. Lubel, Maximal sized antichains in partial oeders, Discrete Math., 1 (1971), 47-53.

[9] D. Kleitman, On an extremal property of antichains in partial orders. The LYM property and some of its implications and application, Combinatorics, Part 2, Math. Center Tracts, No. 56, Math. Centrum Amsterdam, 1974, pp. 77-90.

[10] K.W. Lih, Sperner families over a subset, J. Combin. Theory Ser.A, 29 (1980), 182-185.

[11] Y. Wang, On a class of subspace lattice, J. Math. Res. Expo., 19 (1999), 341-348.

Received: May 23, 2006 